\title{
A method to calculate zero-signature satellite laser ranging normal points for millimeter geodesy - a case study with Ajisai
}

\author{
Daniel Kucharski ${ }^{*}$, Georg Kirchner ${ }^{2}$, Toshimichi Otsubo ${ }^{3}$ and Franz Koidl ${ }^{2}$
}

\begin{abstract}
High repetition-rate satellite laser ranging (SLR) offers new possibilities for the post-processing of the range measurements. We analyze 11 years of $\mathrm{kHz}$ SLR passes of the geodetic satellite Ajisai delivered by Graz SLR station (Austria) in order to improve the accuracy and precision of the principal SLR data product - normal points. The normal points are calculated by three different methods: 1 ) the range residuals accepted by the standard 2.5 sigma filter, 2) the range residuals accepted by the leading edge filter and 3 ) the range residuals given by the single corner cube reflector (CCR) panels of Ajisai.

A comparison of the statistical parameters of the obtained results shows that the selection of the range measurements from the leading edge of the SLR data distribution allows to minimize the satellite signature effect and to reduce the average single-shot RMS per normal point from 15.44 to $4.85 \mathrm{~mm}$. The optical distance between the leading edge mean reflection point and the satellite's center of mass is $1,023 \mathrm{~mm}, \mathrm{RMS}=1.7 \mathrm{~mm}$.

Further, in addition, we utilize the complete attitude model of Ajisai during the post-processing which enables selection of the range measurements to the single CCR panels of the satellite and the formation of the normal points which most closely approximate the physical distance between the ground station and the center of mass of Ajisai. This method eliminates the satellite signature effect from the distribution of the post-fit range residuals and further improves the average single-shot RMS per normal point to $3.05 \mathrm{~mm}$. The normal point RMS per pass is reduced from 2.97 to $0.06 \mathrm{~mm}$ - a value expected for a zero-signature satellite.
\end{abstract}

Keywords: Satellite laser ranging; Ajisai; Satellite geodesy; Zero-signature satellite; Normal point

\section{Background}

\section{Experimental geodetic satellite - Ajisai}

The Experimental Geodetic Satellite Ajisai (Figure 1) was launched on 12 August 1986 by the National Space Development Agency of Japan (NASDA), currently reorganized as the Japan Aerospace Exploration Agency (JAXA). Objective of the mission is the accurate position determination of fiducial points on the Japanese Islands. The satellite is equipped with 1,436 corner cube reflectors (CCR) for satellite laser ranging (SLR), arranged in the form of 15 rings around the symmetry axis. Ajisai is also equipped with 318 mirrors, used in the past to determine the direction to the satellite (Sasaki and Hashimoto 1987).

\footnotetext{
* Correspondence: daniel@drdk.org

${ }^{1}$ Space Science Division, Korea Astronomy and Space Science Institute, 776, Daedeok-Daero, Daejeon 305-348, Yuseong-Gu, Republic of Korea Full list of author information is available at the end of the article
}

The mirrors have also been used for photometric measurements of Ajisai spin (Otsubo et al. 1998). The satellite is placed in a quasi-circular orbit of altitude $1,490 \mathrm{~km}$ and inclination of $50^{\circ}$.

The positions of the $120 \mathrm{CCR}$ panels are presented on Figure 1. Each panel contains 12 CCRs except for the one mounted on the central ring - with 8 CCRs only. The system elements of the satellite are presented on Figure 2. The corner cube reflectors are made of fused silica for 532-nm green light, and the back faces of the reflectors are un-coated.

Ajisai was launched with an initial spin period of $1.5 \mathrm{~s}$ (Hashimoto et al. 2012) and is currently the fastest spinning SLR satellite in orbit. The specific construction of the spherical body (laminated sheets of aluminum foil) minimizes the interaction with the Earth's magnetic field and prevents loss of the rotational energy. The orientation 

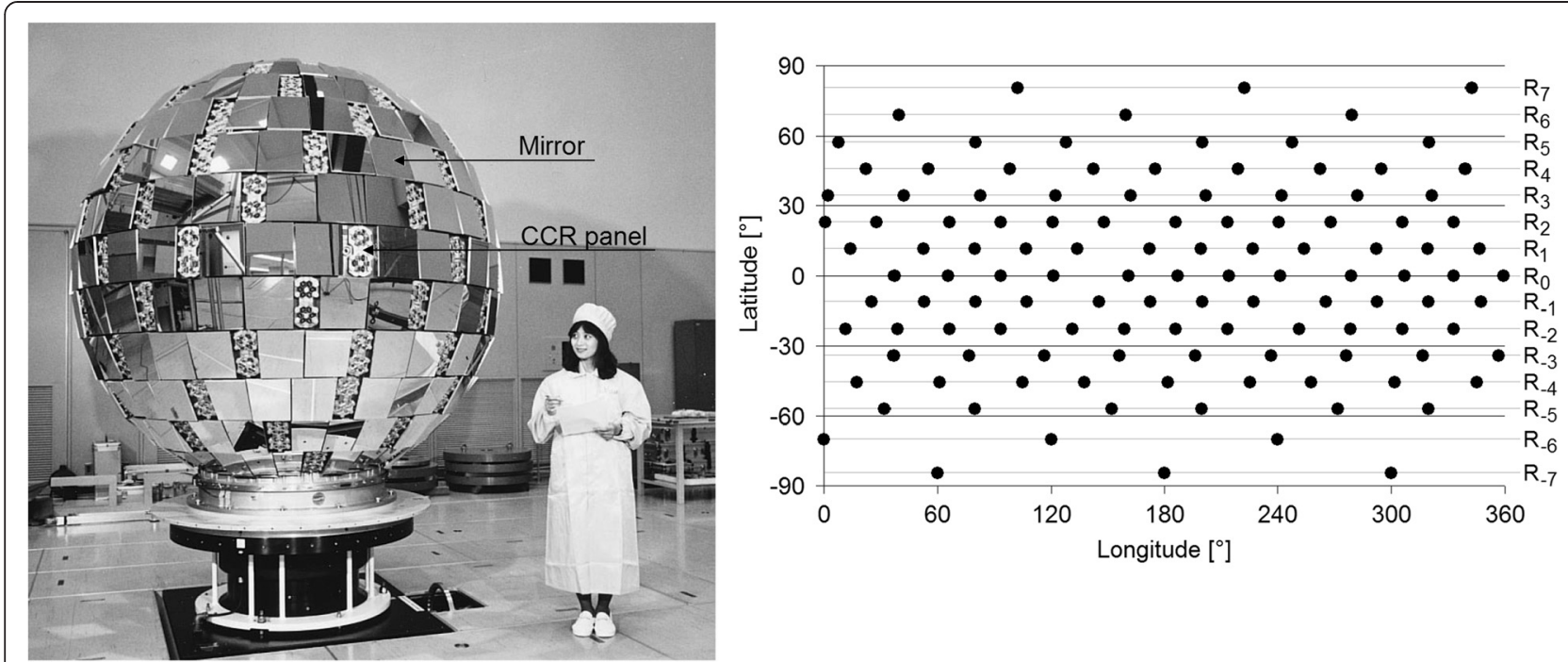

Figure 1 Ajisai (courtesy of JAXA) and positions of the CCR panels on the surface. The retroreflector array consists of 15 rings $R_{-7} \ldots R_{7} ; R_{0}$ is the equator ring.

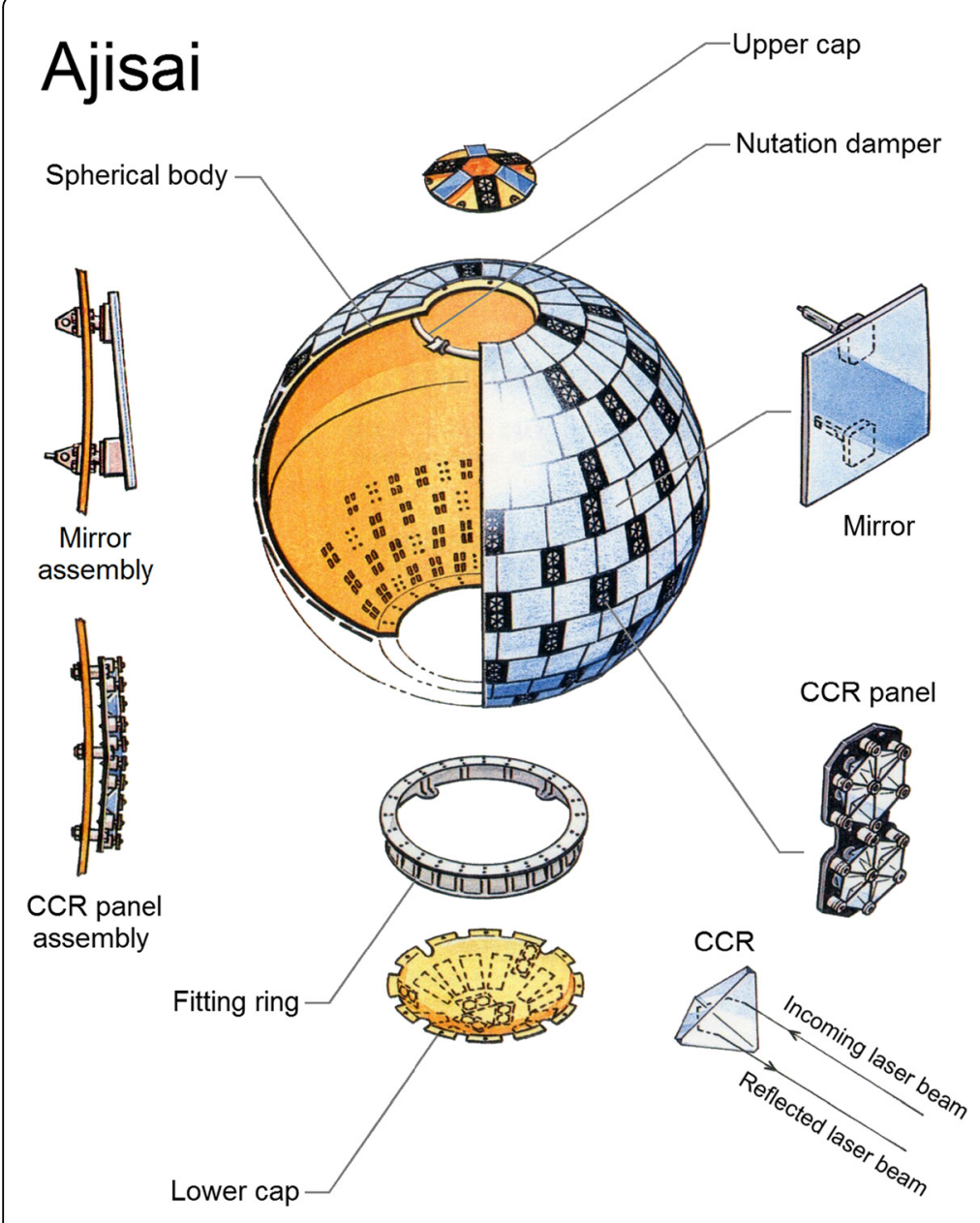

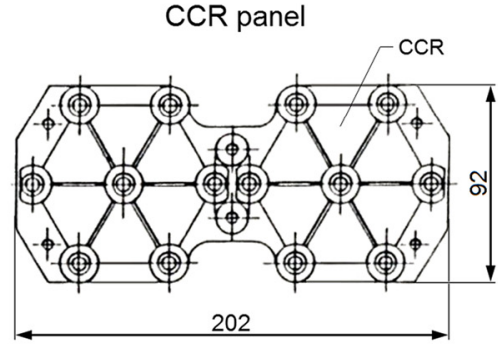

Central axis
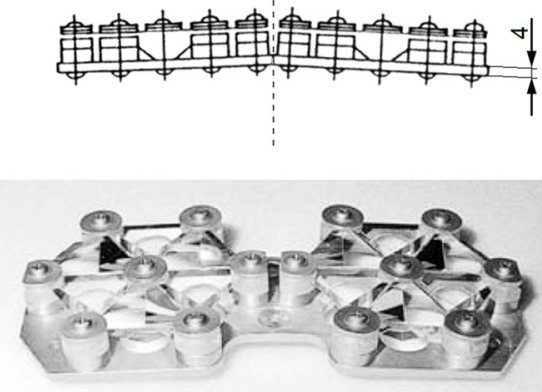

CCR
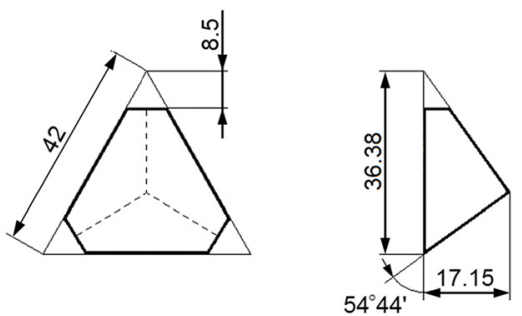

Figure 2 System elements of Ajisai (Hashimoto et al. 2012). 
of the spacecraft was set to be parallel to the Earth's spin axis and is stabilized by the passive nutation dumper (Figure 2).

\section{Satellite laser ranging}

Satellite laser ranging (SLR) measures the distance to satellites equipped with retroreflectors. The laser pulses transmitted from the SLR station are reflected by the spacecraft back to the receiver telescope of the ground system. The time-of-flight measurements of the laser pulses are recalculated into an optical range to the satellite. The range measurements are used for precise orbit determination (POD), study of tectonic plate motion, as well as determination of Earth's gravity field, Earth rotation parameters, and geocenter position (Moore and Wang 2003). The SLR data can be used for deriving the product GM of the universal gravitational constant G with the mass M of the Earth (Dunn et al. 1999).

During a pass of a satellite over an SLR station, the laser range measurements are collected. After the pass, the predicted range trend is fitted to the measured range values by adjusting the time bias and range bias estimations. As the next step, the range residuals are formed by calculating the difference between the observed and predicted (computed) range values, $\mathrm{O}-\mathrm{C}$. In the course of this process, fitting functions (orbital function or low degree polynomials) are used in order to remove the systematic trends from the distribution of the range residual data. The final step of the post-processing is the formation of the so-called normal points (NP).

\section{Normal point formation with sigma filter}

The development of the SLR technology and the use of the 10 to $15 \mathrm{~Hz}$ repetition rate lasers significantly increased the amount of the range measurements per pass compared to the early SLR systems. In order to reduce the computer time required for the orbital analysis of the large data sets, while retaining the information content of the individual range measurements, a data compression technique has been introduced to create normal points. At the 5th Laser Ranging Instrumentation Workshop held at Herstmonceux in 1984, a procedure was recommended for the formation of normal points from the full rate ranging data (Recommendation 84A: 'On SLR normal-point generation and data exchange'). The calculated range residuals of the pass are clipped with an iterative sigma rejection filter. The retained data set is divided into short time slots ( $30 \mathrm{~s}$ for Ajisai); the arithmetic mean of the range residuals of each bin is calculated and used as the reference for the normal point.

The on-site produced normal points are the principal SLR data product and express the one-way optical distance between the reference point of the SLR ground station and the mean reflection point of the satellite (Figure 3A).

In order to deliver an effective distance between the ground SLR system and the satellite's center of mass (which represents its orbital motion), the normal points are corrected for the systematic errors such as the atmospheric refraction delay (Mendes and Pavlis 2004), the calibration system delay, and for the center-of-mass offset. The center-of-mass correction (CoM correction -

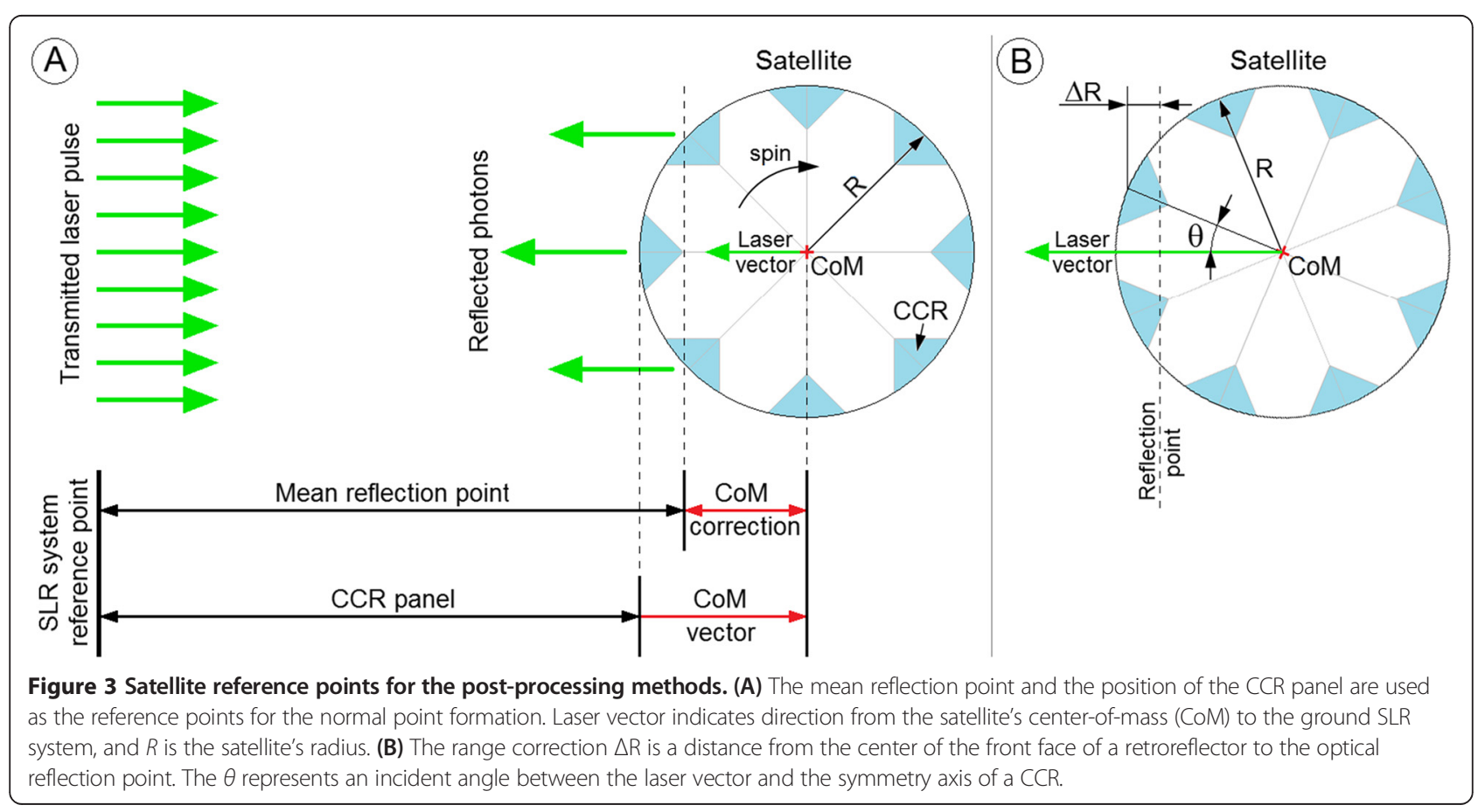


Figure $3 \mathrm{~A}$ ) is the one-way distance to be added to a normal point at the precise orbit determination stage and can be determined from the prelaunch measurements or by the analysis of a theoretical satellite's response function (Arnold 1979). The prelaunch tests give the centerof-mass correction for Ajisai of 1,010 mm (Sasaki and Hashimoto 1987). At the 9th International Laser Ranging Workshop in Canberra in 1994, papers were presented by J. J. Degnan and by R. Neubert which theoretically investigated the reflection signature of the LAGEOS-type satellites and calculated the correction from the mean reflection point to the center of mass of the spherical satellites.

Otsubo and Appleby (2003) analyzed the temporal spread of the optical pulse signals due to a reflection from the multiple onboard reflectors and modeled the response function of LAGEOS, Ajisai, and the Etalons. The obtained center-of-mass corrections depend on the ranging system configuration and observation policy at the terrestrial stations and vary by about $1 \mathrm{~cm}$ for LAGEOS and $5 \mathrm{~cm}$ for Ajisai and Etalons. Otsubo et al. (2000) demonstrated that the center-of-mass correction for Ajisai depends on the orientation of the satellite and can vary between 985 and 1,030 $\mathrm{mm}$ for a Gaussian profile of the incoming laser pulse with FWHM (full width at half maximum) of $200 \mathrm{ps}$.

The optical range measured by SLR is longer from the physical value due to the delay of a laser pulse passing through the glassy corner cube reflectors. The optical distance to a CCR refers to the optical reflection point and can be corrected by the range correction $\Delta \mathrm{R}$ in order to represent the physical distance to the center of the CCR's external surface. The range correction $\Delta \mathrm{R}$ is defined as $\Delta R=L \sqrt{n^{2}-\sin ^{2} \theta}$, where $L$ is the height of CCR (17.15 mm for Ajisai), $n$ is the refractive index of the material (1.46 for fused silica and 532-nm wavelength) and $\theta$ is the incident angle between the laser vector (vector from the satellite's center directed towards the ground SLR system) and the optical axis of a retroreflector (Fitzmaurice et al. 1977, Figure 3B).

In this paper, we propose a 'reflector filter' which allows us to calculate a physical distance to the single CCR panels of Ajisai. The physical range values are corrected by the center-of-mass vector (vector length along the laser range direction $=R \cdot \cos (\theta)$ ) in order to determine the distance between the ground SLR system and the satellite's center of mass (Figure 3A).

\section{SLR measurements to Ajisai at Graz observatory}

Since 8 October 2003, the Graz SLR station (Austria) operates with a $2-\mathrm{kHz}$ repetition-rate $\mathrm{Nd}$ :Van laser of 10ps FWHM pulse duration (Kirchner and Koidl 2004). The detection system is based on the Compensated Single
Photoelectron Avalanche Detector (C-SPAD) (Kirchner and Koidl 1995) and responds to the first incoming photon. The energy-dependent range bias of C-SPAD is reduced to about 10 ps (time walk compensation), thus the measured range does not depend on the energy of the incoming signal.

Figure 4 presents the range residuals of Ajisai obtained by the Graz SLR system at different configuration stages. The three data samples mark the improvements in the amount and the accuracy of the range measurements. The example Figure 4A was measured on 23 August 1986 (11 days after Ajisai was launched) with $2.5-\mathrm{Hz}$ repetition-rate laser - the small amount of the data points gives RMS of $36.8 \mathrm{~mm}$. The case B shows the measurements obtained on 17 January 1996 with the $10-\mathrm{Hz}$ repetition-rate laser. The increase in the amount of measurements per pass improves the RMS $(11.8 \mathrm{~mm})$ and allows for the analysis of the statistical parameters of the data distribution. The $2-\mathrm{kHz}$ repetition-rate laser (case C: pass measured on 17 January 2006) greatly increased the number of the range measurements per second. The high amount of the accurate data and the very short laser pulse length not only improve the statistics of the obtained range residuals (RMS $=8.81 \mathrm{~mm}$ ) but also allow to distinguish between the laser echoes given by the single CCR panels of the satellite (Figure 4C: visible as the down-peaks of the data points).

Figure $5 \mathrm{~A}$ presents a $5 \mathrm{~s}$ part of the Ajisai pass measured by Graz on 17 January 2006. The selected data span shows the range measurements to the CCR panels of the ring $\mathrm{R}_{-3}$ (Figure 1), and the observed $\mathrm{mm}$-scale modulation of the range residuals (amplitude of approximately $25 \mathrm{~mm}$ ) is caused by the rotation of the satellite (spin period of $1.995 \mathrm{~s}$ ).

The presented range residuals are determined during an iterative 2.5 sigma elimination process and give RMS of $8.81 \mathrm{~mm}$. The statistical parameters of the range residual distribution can be obtained with the smoothing algorithm derived by Sinclair (1993). The distribution function is calculated with a strong smoothing coefficient of $15 \mathrm{~mm}$ which assures that all of the leading range residuals will be used during the process of normal point formation. The determined histogram function (Figure 5B) gives peak $=-0.78 \mathrm{~mm}$, full width at half maximum $(\mathrm{FWHM})=41.7 \mathrm{~mm}$, and leading edge at half maximum $(\mathrm{LEHM})=-21.2 \mathrm{~mm}$; the determined leading edge corresponds to the front face of the spherical satellite.

Due to the fast spin of Ajisai, the distance between the nearest CCR panel and the satellite's front face is quickly changing - this effect is represented as the $\mathrm{V}$-shape distribution of the laser echoes on Figure 5C. The situation when the incident angle $\theta$ between the laser vector and the central axis of the panel (Figure 3B) is the smallest 


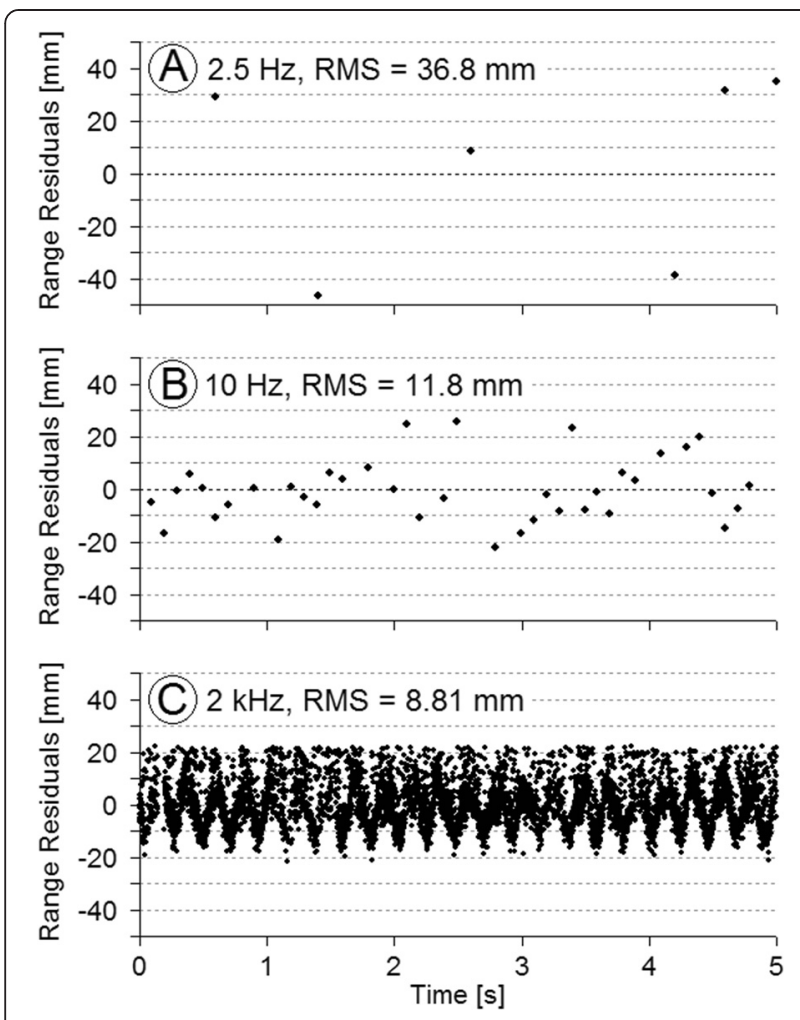

Figure 4 Five seconds parts of Ajisai passes measured by Graz SLR system. Measurements collected on: (A) 23 August 1986, (B) 17 January 1996, (C) 17 January 2006. The laser repetition rate and RMS of the range residuals are given. The 0 level represents the mean of the range residuals.

defines the minimum deviation of the observed CCR panel (MD). The epoch times of the minimum deviation events throughout the pass can be predicted by the complete attitude model of Ajisai. The spin axis orientation of the satellite (in J2000.0 celestial reference frame) during a single pass can be predicted with RMS of $0.128^{\circ}$ (Kucharski et al. 2010b, 2013a). The spin period and the rotational phase angle of Ajisai can be obtained during the post-processing of a complete pass with RMS of $84 \mu \mathrm{s}(42 \mathrm{ppm})$ for the spin period and with RMS of $0.48^{\circ}$ for the phase angle (Kucharski et al. 2010a).

The range coordinate of the minimum deviation (MD) can be calculated as an arithmetic mean of the range residuals located around the predicted MD epoch (data bin width of $20 \mathrm{~ms}$ ) and clipped by an iterative 2.2 sigma filter. In the example case (Figure $5 \mathrm{C}$ ), the range coordinate of the MD event is calculated as the mean of 23 selected points (return rate of 57.5\%) and is equal to $-11.7 \mathrm{~mm}$, RMS $=2.29 \mathrm{~mm}$.

\section{Methods}

Normal point formation with leading edge filter

The standard normal point algorithm was prepared before the effects of the satellite signature were fully recognized. Since the position of the mean reflection point (its distance to the satellite's center of mass) can vary depending on the optical response of the retroreflector array, the further theoretical investigation was done (Appleby 1993, 1995, Neubert 1995, Sinclair et al. 1995) to find the center-of-mass corrections for a variety of the reference points: mean, peak, leading edge at half maximum (LEHM) (Figure 5).

The large amount of data delivered by the high repetition-rate SLR system allows to find a stable leading edge level of the range residual distribution (Figure 5 LEHM) and to use only the leading data points located between the peak and LEHM for the normal point formation. It was demonstrated by Kirchner et al. (2008) that selecting only the leading range measurements significantly improves the stability of the normal points.

In order to compare different post-processing methods presented in this paper, we modify the initial definition

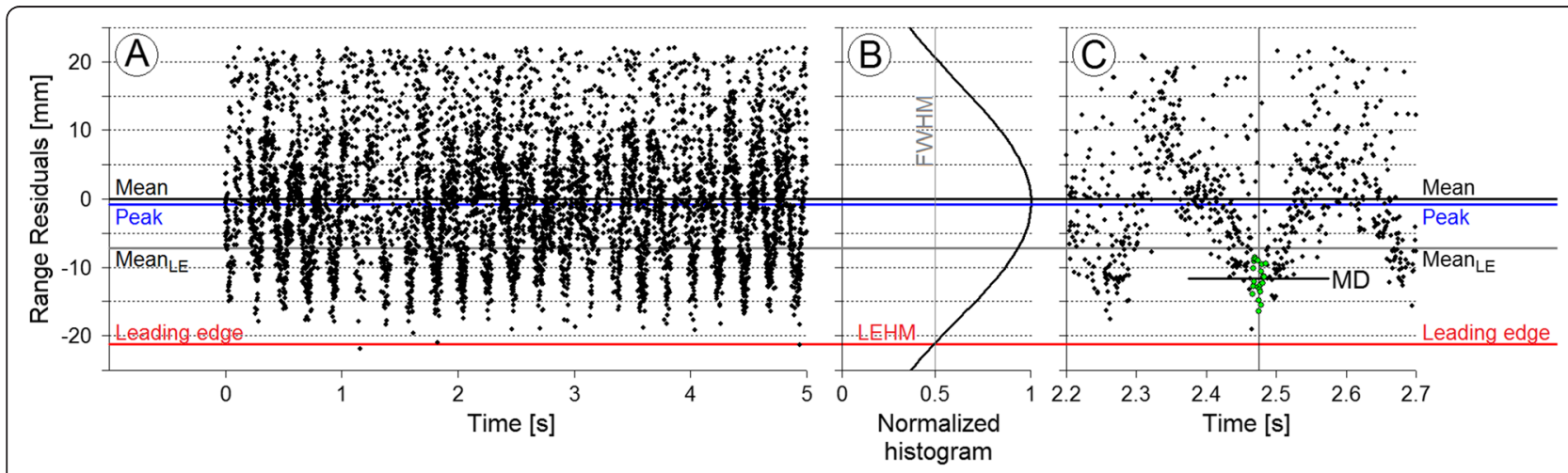

Figure 5 Range residuals of kHz SLR pass. (A) Five seconds part of Ajisai pass measured by Graz on 17 January 2006: 6,337 data points, RMS = $8.81 \mathrm{~mm}$. (B) Histogram of the range residual distribution (obtained with $15-\mathrm{mm}$ smoothing coefficient). (C) V-shape data peak represents change of the distance to the single CCR panel due to the spin of the satellite; the minimum deviation MD of the panel is indicated. The significant levels of the data distribution are marked: mean of the range residuals after 2.5 sigma clipping, peak, mean of the leading edge points (mean $\mathrm{LE}_{\mathrm{E}}$, leading edge at half maximum (LEHM). The full width at half maximum (FWHM) is $41.7 \mathrm{~mm}$. 


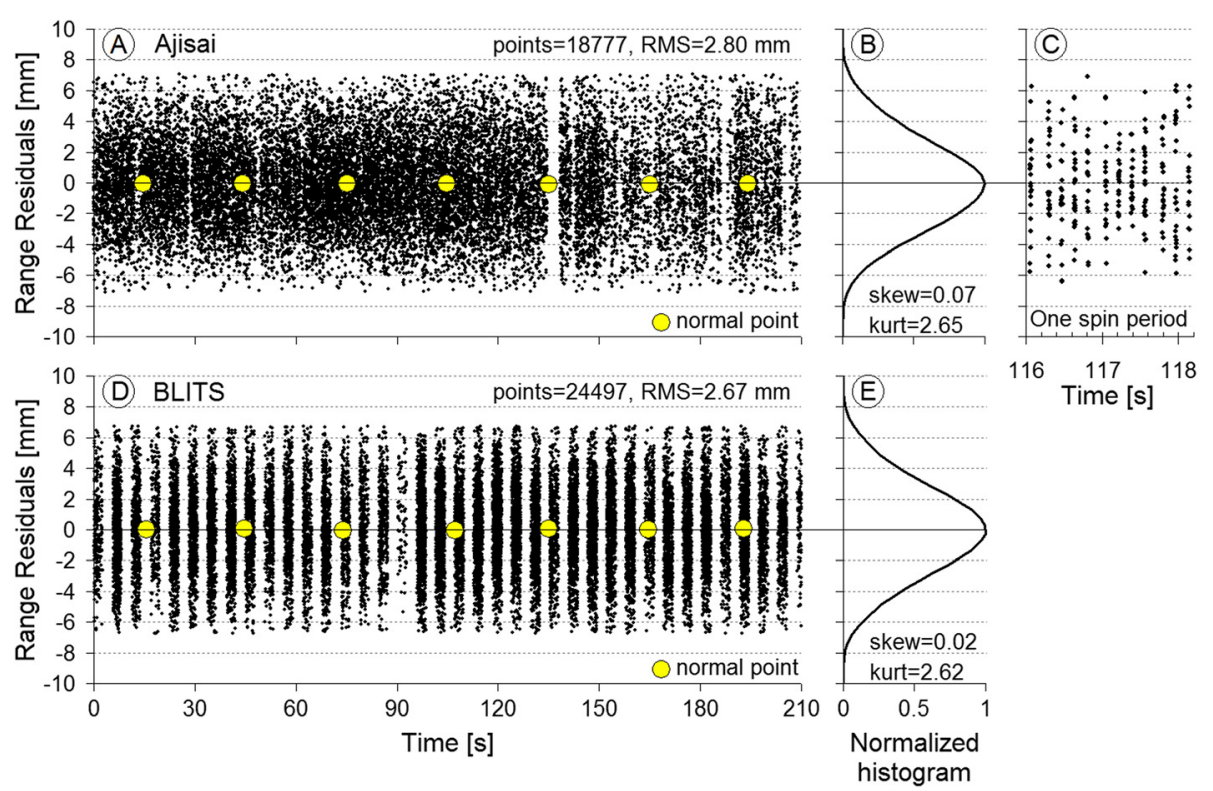

Figure 6 Range residuals and normal points of Ajisai and BLITS. (A) Ajisai, pass measured by Graz on 13 March 2014, and (D) BLITS, pass measured by Graz on 24 January 2012. (B,E) Distribution of the range residuals obtained with a 1-mm smoothing coefficient. (C) Range residuals of Ajisai during one rotation of the satellite (approximately $2.2 \mathrm{~s}$ ). Zero level is the mean of the range residuals.

of the leading edge filter given by Kirchner et al. (2008). The initial algorithm defines the reference for the normal points as a polynomial function fitted to the $10 \%$ of the nearest range residuals. The normal points are formed from the range residuals which lay between the fixed and arbitrary set limits of -3 to $17 \mathrm{~mm}$ from the polynomial function. The modification of the procedure introduced here sets the acceptance levels at the peak and LEHM of the data distribution given by the smoothing algorithm (with the smoothing coefficient of $15 \mathrm{~mm}$, Figure $5 \mathrm{~B}$ ). Thus, the mean reflection point calculated with the leading edge filter (Figure $5 \mathrm{~A}$ : mean $_{\mathrm{LE}}$ level) is located on the leading slope of the data distribution.

\section{Normal point formation with reflector filter}

For a given $\mathrm{kHz}$ SLR pass, the spin parameters of Ajisai are determined and the epochs of the minimum deviation events throughout the pass are predicted (Kucharski et al. 2010a). The range residuals around each predicted minimum deviation epoch (within \pm 10 ms from an MD epoch) are selected and clipped by an iterative 2.2 sigma filter. If the amount of the remaining data points is $\geq 10$ (return rate of $25 \%$ ) and the RMS is $\leq 5 \mathrm{~mm}$, then the selected range residuals are considered to be given by a single CCR panel and their mean represents the optical distance to the given panel (Figure 5C: minimum deviation MD level). In order to determine the physical distance to the identified panel, the selected range residuals and the corresponding range measurements are corrected by the range correction $\Delta \mathrm{R}$ (Figure $3 \mathrm{~B}$ ). $\Delta \mathrm{R}$ varies from 25.04 to 25.02 for the incident angle $\theta$ between the laser vector and the symmetry axis of a CCR panel from $0^{\circ}$ to $3^{\circ}$. The corrected data points refer to the external surface of the identified CCR panel which is placed on the radial distance of $R=$ $1,053 \mathrm{~mm}$ from the satellite's center of geometry (coincides with the center of mass). As the final step, the center-of-mass vector (Figure 3A) is added to each of the selected data points and the mean of the resulting range residuals indicates position of the satellite's center of mass (Figure 6).

This process is applied to the predicted minimum deviation events if the incident angle $\theta$ between the laser beam vector and the central axis of a panel is $\leq 3^{\circ}$. This arbitrarily set limit is related to the complex structure of

Table 1 The mean values of the parameters calculated from 2,731 passes of Ajisai and 450 passes of BLITS

\begin{tabular}{llll}
\hline & Unit & Ajisai & BLITS \\
\hline Pass RMS of range residuals (after 2.5 sigma filtering) & $\mathrm{mm}$ & $3.04 \pm 0.29$ & $3.17 \pm 0.31$ \\
Normal point RMS per pass & $\mathrm{mm}$ & $0.06 \pm 0.02$ & $0.09 \pm 0.05$ \\
Single-shot RMS per normal point & $\mathrm{mm}$ & $3.05 \pm 0.36$ & $3.21 \pm 0.32$ \\
Return rate per normal point & $\%$, points & $1.6 \%, 960$ & $5.8 \%, 3,480$ \\
\hline
\end{tabular}


the Ajisai CCR panel (Figure 2 - right: CCR panel) and assures that during the minimum deviation event, the SLR system receives the laser echoes in equal proportions from the two sides of the panel. Identification of the laser echoes given by the single CCR panels allows to refer the particular range measurements to the satellite's center of mass and eliminates the satellite signature effect from the normal points.

The zero-signature SLR data of Ajisai can be compared with the range residuals of the spherical glass retroreflector satellite BLITS (Vasiliev et al. 2007). The satellite represents the concept of a Luneburg lens and consists of an inner glass sphere covered by the concentric glass half-shells of which one is aluminum coated. BLITS acts as a single retroreflector and thus it is the zero-signature satellite (Kucharski et al. 2011a, b). The mission started on 17 September 2009 and ended after the possible collision with a space debris on 22 January 2013 (Parkhomenko et al. 2013).

Figure 6 presents the zero-signature range residuals of Ajisai (Figure 6A) and BLITS (Figure 6D) measured by Graz SLR system on 13 March 2014 and 24 January 2012, respectively. The selected time span of 210 s corresponds to the duration of seven normal points. The range residuals are processed by an iterative 2.5 sigma filter and give RMS of $2.8 \mathrm{~mm}$ for Ajisai and $2.67 \mathrm{~mm}$ for BLITS. The specific construction of BLITS makes the range measurements possible only when the transparent hemisphere of the lens is visible from the SLR station. Due to the spin of the satellite (Kucharski et al. 2013b), the laser beam of the SLR system is alternately pointing to the transparent and the coated hemisphere, thus series of measurement/no measurement intervals during a pass are visible (Figure 6D). Figure 6C presents the selected range residuals during one rotation of Ajisai (approximately $2.2 \mathrm{~s}$ ).

Processing of the large amount of $\mathrm{kHz}$ SLR passes measured by Graz allows to compare the key parameters of the Ajisai and BLITS data distribution. We have analyzed 2,731 passes of Ajisai measured from 15 October 2003 until 8 September 2014 and 450 passes of BLITS measured from 27 September 2009 until 18 June 2012; the obtained parameters are presented in Table 1.

The mean pass RMS of the range residuals of Ajisai and BLITS is at the level of $3 \mathrm{~mm}$ (obtained with 2.5 sigma filter) and coincides with the RMS of the SLR system calibration to the ground target of $2.25 \mathrm{~mm}$ (obtained with 2.2 sigma filter). The RMS of BLITS range residuals is slightly larger than the value of Ajisai; the complex structure of the spherical lens reflector (two kinds of glass with different refractive indices) can affect the shape of the retroreflected laser pulse. The symmetrical distribution of the range residuals around the 0 level (Figure 6A,D) gives a very low normal point RMS per pass: $0.06 \mathrm{~mm}$ for Ajisai and $0.09 \mathrm{~mm}$ for BLITS.

The low, average return rate of $1.6 \%$ for Ajisai normal points is caused by the $3^{\circ}$ limit of the maximum incident angle $\theta$ between the laser beam vector and the central axis of a CCR panel. The average number of the range measurements per normal point can be increased from the present 960 by widening the $\theta$ incident angle limit or by increasing the repetition rate of the laser from present 2 to $10 \mathrm{kHz}$ (while maintaining the return rate at the high level).

\section{Results}

In this work, we have calculated normal points using three different post-processing methods: 1) the range residuals accepted by the standard 2.5 sigma filter (designated as $\mathrm{NP}_{\mathrm{S}}$ ), 2) the range residuals accepted by the leading edge filter $\left(\mathrm{NP}_{\mathrm{LE}}\right)$ and 3$)$ the range residuals given by the single $\mathrm{CCR}$ panels $\left(\mathrm{NP}_{\mathrm{CCR}}\right)$. The first two methods refer to the optical mean reflection point of the retroreflector array while the third method - to the physical position of the satellite's center of mass (Figure 3A). The obtained single-shot RMS values per normal point are presented on Figure 7. The mean single-shot RMS per $\mathrm{NP}_{\mathrm{S}}$ is $15.44 \pm 5.23 \mathrm{~mm}, \mathrm{NP}_{\mathrm{LE}}: 4.85 \pm 0.9 \mathrm{~mm}$, and $\mathrm{NP}_{\mathrm{CCR}}$ : $3.05 \pm 0.36 \mathrm{~mm}$. The linear approximation of the RMS values presents the following dependence on the satellite's elevation: $\mathrm{NP}_{\mathrm{S}}$ : $-0.072 \mathrm{~mm} /{ }^{\circ}, \mathrm{NP}_{\mathrm{LE}}:-0.013 \mathrm{~mm} /{ }^{\circ}$, and $\mathrm{NP}_{\mathrm{CCR}}:-0.005 \mathrm{~mm} /{ }^{\circ}$. Average $\mathrm{NP}_{\mathrm{S}} \mathrm{RMS}$ per pass is
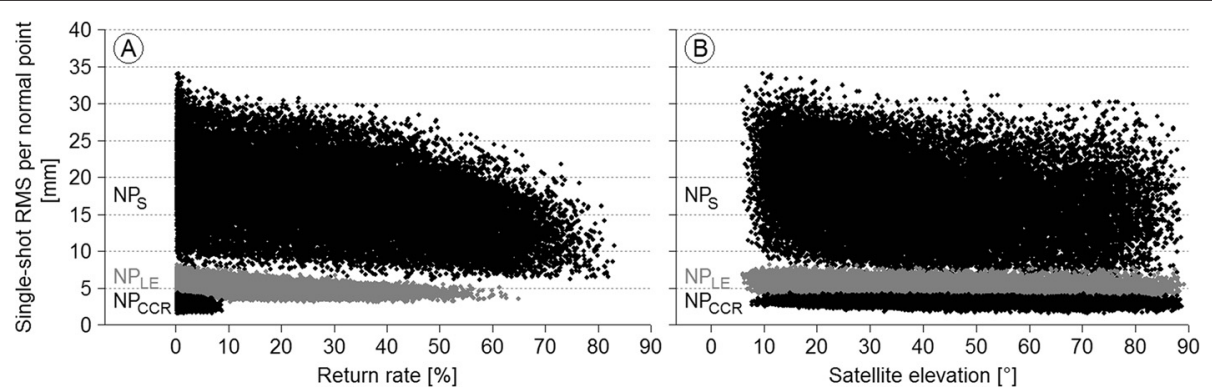

Figure 7 Single-shot RMS per normal point. Data obtained by three post-processing methods: 2.5 sigma clipping $\left(N P_{S}\right)$, leading edge filter ( $\left.N P_{L E}\right)$, reflector filter $\left(\mathrm{NP}_{\mathrm{CCR}}\right)$. (A) Single-shot RMS per normal point versus return rate, (B) Single-shot RMS per normal point versus satellite elevation. 
Table 2 Distance between the significant levels of the range residual distribution (Figure 5) and Ajisai center of mass

\begin{tabular}{llll}
\hline & Physical distance to CoM [mm] & Optical distance to CoM [mm] & RMS [mm] \\
\hline Minimum deviation MD & 1,052 & 1,027 & 0.4 \\
NP $_{\text {LE }}$ (mean LE level on Figure 5) & 1,048 & 1,023 & 1.7 \\
Peak & 1,041 & 1,016 & 2.6 \\
NPS (after 2.5 sigma clipping) (Mean level on Figure 5) & 1,037 & 1,012 & 4.7 \\
\hline
\end{tabular}

The physical distance between the outer surface of the CCRs and the satellite's center of mass is $1,053 \pm 5 \mathrm{~mm}$.

$2.97 \pm 0.9 \mathrm{~mm}, \mathrm{NP}_{\mathrm{LE}}: 0.49 \pm 0.21 \mathrm{~mm}$, and $\mathrm{NP}_{\mathrm{CCR}}: 0.06 \pm$ $0.02 \mathrm{~mm}$. The limit of $3^{\circ}$ for the maximum incident angle $\theta$ between the laser beam and the central axis of the CCR panel reduces the average number of normal points per pass from 20.9 $\mathrm{NP}_{\mathrm{S}} / \mathrm{NP}_{\mathrm{LE}}$ to $8.9 \mathrm{NP}_{\mathrm{CCR}}$. The number of $\mathrm{NP}_{\mathrm{CCR}}$ per pass can be increased if the specific structure of the Ajisai CCR panels is taken into account for the higher incident angle $\theta$.

The minimum deviation level (MD on Figure $5 \mathrm{C}$ ) indicates the position of the specific CCR panel, thus it can be used as the reference to calculate the distance between the satellite's center of mass and the significant levels of the range residuals distribution: peak, LEHM, $\mathrm{NP}_{\mathrm{S}}$ (refers to mean on Figure 5), and $\mathrm{NP}_{\mathrm{LE}}$ (refers to mean $_{\mathrm{LE}}$ on Figure 5).

For every minimum deviation event, the normal points $\mathrm{NP}_{\mathrm{S}}$ and $\mathrm{NP}_{\mathrm{LE}}$ are calculated (range residuals selected from a data span of $\pm 15 \mathrm{~s}$ around the predicted MD epoch). The results obtained from analysis of 2,731 Ajisai passes (1,468,745 minimum deviation events) are presented in Table 2. The mean FWHM of the pass range residual distribution (after 2.5 sigma clipping) is $50.19 \mathrm{~mm}, \mathrm{RMS}=7.87 \mathrm{~mm}$, and the mean width of the leading edge band (distance between peak and LEHM Figure 5A) is $21.89 \mathrm{~mm}$, RMS $=1.74 \mathrm{~mm}$.

The optical distance to the satellite's center of mass presented in Table 2 is shorter than the physical distance by the range correction value $(\Delta \mathrm{R}=25.04 \mathrm{~mm})$ calculated for the incident angle $\theta=0^{\circ}$ (Figure 3B). The optical distance between the mean (reference level for $\mathrm{NP}_{\mathrm{S}}$ ) and CoM is $1,012 \mathrm{~mm}$ and coincides with the standard center of mass correction of $1,010 \mathrm{~mm}$ (Otsubo and Appleby 2003). The RMS of the mean LE$-\mathrm{CoM}$ distance $(1.7 \mathrm{~mm})$ is significantly lower than the RMS of the mean-CoM $(4.7 \mathrm{~mm})$, thus the mean $_{\mathrm{LE}}$ can serve as a more stable reference level for the normal points.

\section{Discussion}

The leading edge post-processing method reduces the satellite signature effect and gives a more stable reference level $\left(\operatorname{mean}_{\mathrm{LE}}\right)$ for the normal points. This method is based on the standard routines and can be easily implemented in the on-site post-processing software. Graz SLR station uses the leading edge filter to compute the normal points of Ajisai since 5 March 2008 and of Etalon-1, Etalon-2, LAGEOS-1, and LAGEOS-2 since 5



Figure 8 Sixty seconds part of LARES pass measured by Graz SLR system on 11 March 2014. Moving average is plotted, RMS = 2.91 mm (after 2.5 sigma clipping). Zero level indicates the mean of the post-fit range residuals. 
February 2008. It should be noted that the use of the mean $_{\mathrm{LE}}$ as the reference for the normal points must be followed by a proper center-of-mass correction in the course of the POD analysis.

The reflector filter eliminates the satellite signature effect from the distribution of the post-fit range residuals and enables the computation of the normal points which represent the physical distance to the satellite's center of mass at the ultimate accuracy level.

It is important to note that approximately $10 \mathrm{~mm}$ offset error in the laser range measurements can cause a few ppb error in the determination both of the value of GM and in the scale of the terrestrial reference frame. The zero-signature normal points of the geodetic satellites delivered by the globally distributed SLR systems (Pearlman et al. 2002) could improve the accuracy of the precise orbit determination and help to achieve the GGOS geodetic reference frame requirements (Plag and Pearlman 2009).

The reflector filter can be applied to the $\mathrm{kHz}$ SLR measurements of any satellite whose complete attitude model is known. The $\mathrm{kHz}$ SLR data of LARES reveals similar to Ajisai - oscillations of the range measurements in the millimeter scale due to the spin of the satellite (Figure 8; Kucharski et al. 2012). Our follow-on research will be devoted to the development of the complete attitude model of LARES and to the generation of the zerosignature normal points from the range measurements provided by the high repetition-rate SLR systems.

\section{Conclusions}

The high repetition-rate SLR technology offers new possibilities for the post-processing of the range observations. The standard clipping process can be improved by the leading edge filter described here which minimizes the satellite signature effect and reduces the average singleshot RMS per normal point from 15.44 to $4.85 \mathrm{~mm}$ (Figure 7). The stable, optical distance between the leading edge mean reflection point (Figure 5: mean $_{\mathrm{LE}}$ ) and the center of mass of Ajisai is $1,023 \mathrm{~mm}$, RMS $=1.7 \mathrm{~mm}$.

High repetition-rate satellite laser ranging efficiently measures the attitude of fully passive, geodetic satellites during day and night. The attitude model of Ajisai applied during post-processing allows to select the range measurements to the single CCR panels and to form normal points which are accurately referenced to the physical distance between the ground station and the satellite's center of mass. This process eliminates completely the satellite signature effect from the distribution of the post-fit range residuals and further improves the average single-shot RMS per normal point to $3.05 \mathrm{~mm}$ (Figure 7). The normal point RMS per pass is reduced from 2.97 to $0.06 \mathrm{~mm}$ - a value expected for a zerosignature satellite.

\section{Competing interests}

The authors declare that they have no competing interests.

\section{Authors' contributions}

The manuscript has been written by DK and reviewed by all authors. GK and FK provided the KHz SLR full rate data of Ajisai for the analysis. GK and TO contributed with their experience in the $\mathrm{kHz}$ SLR technology and in the center-of-mass correction study, respectively. All authors read and approved the final manuscript.

\section{Author details}

${ }^{1}$ Space Science Division, Korea Astronomy and Space Science Institute, 776, Daedeok-Daero, Daejeon 305-348, Yuseong-Gu, Republic of Korea. ${ }^{2}$ Space Research Institute of the Austrian Academy of Sciences, Lustbuehelstrasse 46, A-8042 Graz, Austria. ${ }^{3}$ Hitotsubashi University, 2-1 Naka, Kunitachi 186-8601, Japan.

Received: 13 October 2014 Accepted: 6 February 2015

Published online: 01 March 2015

\section{References}

Appleby GM (1993) Satellite signatures in SLR observations. In: Degnan JJ (ed) Proceedings of 8 Int. Workshop on Laser Ranging Instrumentation. 18-22 May 1993, NASA, Goddard Space Flight Center, Annapolis, MD, USA, pp 2-1-14 Appleby G (1995) Centre of mass corrections for LAGEOS and Etalon for singlephoton ranging systems. In: Sinclair AT (ed) Proceedings of Annual Eurolas Meeting, Munich, Germany, pp 18-26, 20-21 March 1995. Royal Greenwich Observatory, England

Arnold DA (1979) Method of calculating retroreflector-array transfer functions. Special Report 382. Smithsonian Astrophysical Observatory, Cambridge, Massachusetts, USA

Dunn P, Torrence M, Kolenkiewicz R, Smith D (1999) Earth scale defined by modern satellite ranging observations. Geophys Res Lett 26(10):1489-1492, doi:10.1029/1999GL900260

Fitzmaurice MW, Minott PO, Abshire JB, Rowe HE (1977) Prelaunch testing of the Laser Geodynamic Satellite (LAGEOS). NASA Technical Paper, NASA., p 1062

Hashimoto H, Nakamura S, Shirai H, Sengoku A, Fujita M, Sato M, Kunimori H, Otsubo T (2012) Japanese Geodetic Satellite AJISAl: development, observation and scientific contributions to geodesy. J Geodetic Soc Jpn 58 (1):9-25, doi:10.11366/sokuchi.58.9

Kirchner G, Koidl F (1995) Automatic compensation of SPAD time walk effects. In: Sinclair AT (ed) Proceedings of Annual Eurolas Meeting. 20-21 March 1995, Royal Greenwich Observatory, England, Munich, Germany, pp 73-77

Kirchner G, Koidl F (2004) Graz kHz SLR system: design, experiences and results. In: Garate J, Davila JM, Noll C, Pearlman M (eds) Proceedings of 14 Int. Workshop on Laser Ranging, San Fernando, Spain, pp 501-505, 7-11 June 2004

Kirchner G, Kucharski D, Koidl F (2008) Millimeter ranging to centimeter targets. In: Schillak S (ed) Proceedings of 16 Int. Workshop on Laser Ranging, Poznan, Poland, pp 370-372, 13-17 October 2008

Kucharski D, Kirchner G, Otsubo T, Koidl F (2010a) The impact of solar irradiance on Ajisai's spin period measured by the Graz 2 kHz SLR system. IEEE Trans Geosci Remote Sens 48(3):1629-1633, doi:10.1109/TGRS.2009.2031229

Kucharski D, Otsubo T, Kirchner G, Koidl F (2010b) Spin axis orientation of Ajisai determined from Graz 2 kHz SLR data. Adv Space Res 46(3):251-256, doi:10.1016/j.asr.2010.03.029

Kucharski D, Kirchner G, Koidl F (2011a) Spin parameters of nanosatellite BLITS determined from Graz 2 kHz SLR data. Adv Space Res 48(2):343-348, doi:10.1016/j.asr.2011.03.027

Kucharski D, Kirchner G, Lim H-C, Koidl F (2011b) Optical response of nanosatellite BLITS measured by the Graz 2 kHz SLR system. Adv Space Res 48(8):1335-1340, doi:10.1016/j.asr.2011.06.016

Kucharski D, Otsubo T, Kirchner G, Bianco G (2012) Spin rate and spin axis orientation of LARES spectrally determined from satellite laser ranging data. Adv Space Res 50(11):1473-1477, doi:10.1016/j.asr.2012.07.018

Kucharski D, Otsubo T, Kirchner G, Lim H-C (2013a) Spectral response of Experimental Geodetic Satellite determined from high repetition rate SLR data. Adv Space Res 51(1):162-167, doi:10.1016/j.asr.2012.09.018

Kucharski D, Kirchner G, Lim H-C, Koidl F (2013b) New results on spin determination of nanosatellite BLITS from high repetition rate SLR data. Adv Space Res 51(5):912-916, doi:10.1016/j.asr.2012.10.008 
Mendes VB, Pavlis EC (2004) High-accuracy zenith delay prediction at optical wavelengths. Geophys Res Lett 31(14), L14602, doi:10.1029/2004GL020308

Moore P, Wang J (2003) Geocentre variation from laser tracking of LAGEOS1/2 and loading data. Adv Space Res 31(8):1927-1933, doi:10.1016/S0273-1177 (03)00170-4

Neubert R (1995) An analytical model of satellite signature effects. In: McK Luck J (ed) Proceedings of 9 Int. Workshop on Laser Ranging Instrumentation, Canberra, Australia, pp 7-11, November 1995

Otsubo T, Appleby GM (2003) System-dependent center-of-mass correction for spherical geodetic satellites. J Geophys Res 108(B4):2201, doi:10.1029/2002JB002209

Otsubo T, Amagai J, Kunimori H (1998) Measuring Ajisai's spin motion. In: Proceedings of $11 \mathrm{Int}$. Workshop on Laser Ranging, Deggendorf, Germany, pp 674-677, 21-25 September 1998

Otsubo T, Amagai J, Kunimori $\mathrm{H}$, Elphick M (2000) Spin motion of the Ajisai satellite derived from spectral analysis of laser ranging data. IEEE Trans IEEE Geosci Remote Sens 38(3):1417-1424, doi: 10.1109/36.843036

Parkhomenko N, Shargorodsky VD, Vasiliev VP, Yurasov V (2013) Accident in orbit. In: Proceedings of 18 Int. Workshop on Laser Ranging, Fujiyoshida, Japan, pp 13-Po03, 11-15 November 2013

Pearlman MR, Degnan JJ, Bosworth JM (2002) The international laser ranging service. Adv Space Res 30(2):135-143, doi:10.1016/S0273-1177(02)00277-6

Plag HP, Pearlman M (eds) (2009) Global Geodetic Observing System - meeting the requirements of a global society on a changing planet in 2020. SpringerVerlag Berlin, Heidelberg

Sasaki M, Hashimoto H (1987) Launch and observation program of the experimental geodetic satellite of Japan. IEEE Trans Geosci Remote Sensing GE-25(5):526-533, doi:10.1109/TGRS.1987.289830

Sinclair AT (1993) SLR data screening; location of peak of data distribution. In: Degnan JJ (ed) Proceedings of 8 Int. Workshop on Laser Ranging Instrumentation, Annapolis, MD, USA, pp 2-34, 43, 18-22 May 1993

Sinclair AT, Neubert R, Appleby GM (1995) The LAGEOS centre of mass correction for different detection techniques. In: Sinclair AT (ed) Proceedings of Annual Eurolas Meeting, Munich, Germany, pp 31-36, 20-21 March 1995. Royal Greenwich Observatory, England

Vasiliev VP, Shargorodsky VD, Novikov SB, Chubykin AA, Parkhomenko NN, Sadovnikov MA (2007) Progress in laser systems for precision ranging, angle measurements, photometry, and data transfer. Paper presented at the International Laser Ranging Service workshop. The Observatoire de la Cote d'Azur (OCA), France, Grasse, France, 25-28 September 2007

\section{Submit your manuscript to a SpringerOpen ${ }^{\circ}$ journal and benefit from:}

- Convenient online submission

- Rigorous peer review

- Immediate publication on acceptance

- Open access: articles freely available online

- High visibility within the field

- Retaining the copyright to your article

Submit your next manuscript at $>$ springeropen.com 